\title{
Adsorption and two-dimensional phases of a large polar molecule: Sub-phthalocyanine on Ag(111)
}

\author{
S. Berner, ${ }^{1, *}$ M. de Wild, ${ }^{1}$ L. Ramoino, ${ }^{1}$ S. Ivan, ${ }^{2}$ A. Baratoff, ${ }^{1}$ H.-J. Güntherodt, ${ }^{1}$ H. Suzuki, ${ }^{3}$ D. Schlettwein, ${ }^{4}$ \\ and T. A. Jung 5 \\ ${ }^{1}$ National Center of Competence in Research on Nanoscale Science, Department of Physics and Astronomy, University of Basel, \\ Klingelbergstrasse 82, CH-4056 Basel, Switzerland \\ ${ }^{2}$ Institute of Organic Chemistry, University of Basel, St. Johanns-Ring 19, CH-4056 Basel, Switzerland \\ ${ }^{3}$ Kansai Advanced Research Center, C.R.L., 588-2 Iwaoka, Nishi-ku, Kobe 651-2492, Japan \\ ${ }^{4}$ Fachbereich Chemie, University of Oldenburg, 26129 Oldenburg, Germany \\ ${ }^{5}$ Laboratory for Micro- and Nanostructures, Paul Scherrer Institut, CH-5232 Villigen, Switzerland \\ (Received 12 March 2003; revised manuscript received 4 June 2003; published 11 September 2003)
}

\begin{abstract}
The adsorption and the two-dimensional (2D) ordering of chloro[subphthalocyaninato]boron(III) (SubPc) on $\mathrm{Ag}(111)$ has been studied in detail by combined scanning tunneling microscopy and photoelectron spectroscopy at room temperature. SubPc is a polar, highly symmetric molecule, consisting of an extended aromatic system and a central $\mathrm{B}-\mathrm{Cl}$ bond. When growing on $\mathrm{Ag}(111)$ an interesting phase behavior is observed for the first molecular layer of SubPc. At low coverage, below $\approx 0.2$ monolayer $(\mathrm{ML})$, a $2 \mathrm{D}$ lattice gas is present, whereas at medium coverage (on the order of $0.2-0.5 \mathrm{ML}$ ), 2D condensed molecular islands are observed in coexistence with the 2D lattice gas. In these condensed islands, the molecules assemble into a well-ordered honeycomb pattern. At higher coverage (approximately 0.5-0.9 ML) the molecules organize into a 2D hexagonal close-packed (hcp) pattern, in equilibrium with a dense 2D gas phase. In the honeycomb and in the hcp pattern, individual molecules are imaged with submolecular resolution, giving information on their orientation. For both the honeycomb and hcp patterns, islands with two different orientations of the superstructures with respect to the $\operatorname{Ag}(111)$ substrate are observed. In case of the honeycomb pattern, the two superstructures are enantiomorphic. The chirality of these layers originates in the loss of the symmetry of the metal surface upon adsorption of SubPc, while the molecules alone are intrinsically achiral. Based on different photoelectron spectroscopy experiments we conclude that the SubPc molecule is adsorbed on $\mathrm{Ag}(111)$ with its $\mathrm{Cl}$ atom towards the substrate and that the molecule remains intact. Finally, several aspects of the observed 2D condensed phases and the thermodynamic phase behavior are discussed with respect to the charge distribution and the adsorption physics and chemistry of the SubPc molecules.
\end{abstract}

DOI: 10.1103/PhysRevB.68.115410 PACS number(s): 68.43.Hn, 81.07.Nb, 68.37.Ef, 79.60.Dp

\section{INTRODUCTION}

The adsorption physics and chemistry of functional organic molecules on metallic surfaces are of great interest because of their electronic ${ }^{1,2}$ and optic properties. Fundamental efforts to explore and understand these properties of adsorbed molecules are also driven by the emergence of organic semiconductors in technological applications ${ }^{3,4}$ with active volumes shrinking to the nanometer scale, where contact properties can no longer be ignored. Intermolecular and molecule-surface interactions of adsorbates on metal surfaces affect diffusion, island nucleation and growth, and therefore influence the structure and properties of overlayers. ${ }^{5-10}$ Ordered molecular layers can show chirality in the case of chiral $^{11}$ and achiral molecules..$^{10,12,13}$ The latter are formed whenever intermolecular interactions favor arrangements which break the symmetries of the underlying substrate.

Metal phthalocyanines are organic molecules which can be used as functional dyes. ${ }^{14}$ Phthalocyanines were among the first organic molecules to be studied by scanning tunneling microscopy ${ }^{15,16}$ (STM) and were also investigated by means of photoelectron spectroscopy. ${ }^{17,18}$ In the present work, chloro[subphthalocyaninato]boron(III) (SubPc), a polar molecule with an aromatic 14- $\pi$-electron system, has been investigated in detail on $\mathrm{Ag}(111)$. SubPc differs in several ways from the usual fourfold symmetric phthalocya- nines: In the case of SubPc the central metal atom is replaced by boron which is $\mathrm{s}^{3}$ coordinated to an apex chlorine and to three instead of four isoindoline rings. ${ }^{19-23}$ In contrast to the mostly planar phthalocyanines, the SubPc molecule is cone shaped.

The adsorption and growth of SubPc on $\operatorname{Ag}(111)$ in the submonolayer to monolayer regime are investigated. The adsorption chemistry is analyzed by photoelectron spectroscopy, and the arrangement of the molecular layers is investigated by STM at room temperature. The combination of these techniques provides a detailed picture of the coveragedependent molecular adsorption, growth, and twodimensional (2D) phase behavior.

\section{EXPERIMENT}

All experiments were performed in a multichamber ultrahigh-vacuum system, providing different in situ preparation and characterization methods. $\mathrm{Ag}(111)$ films were prepared by evaporation of $\mathrm{Ag}$ onto cleaved mica. ${ }^{24}$ Prior to molecular deposition, the quality of the $\operatorname{Ag}(111)$ substrates was checked by means of STM, x-ray photoelectron spectroscopy (XPS), and low-energy electron diffraction (LEED). From the observed LEED pattern it is apparent that the film grows in the (111) direction but exhibits domains with different rotational orientations. The prepared and characterized 


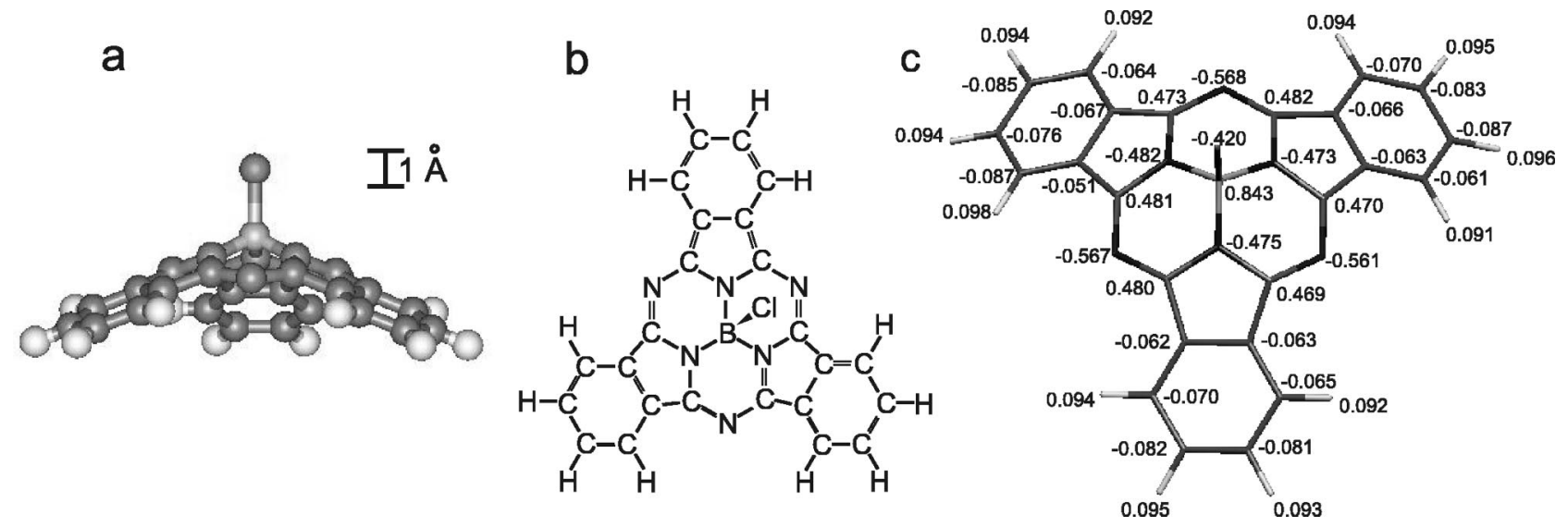

FIG. 1. SubPc molecule: (a) Side view of the calculated geometric structure. The scale bar is valid for (a). (b) Chemical Structure. (c) Effective atomic charges determined by a CHelpG population analysis.

$\operatorname{Ag}(111)$ substrates were used for several experiments in a row. For this purpose molecular layers were removed by cycles of argon ion etching and annealing to $T=570 \mathrm{~K}$ in order to regain atomically clean $\operatorname{Ag}(111)$ surfaces.

The organic molecules were deposited by sublimation from a resistively heated tantalum crucible. During deposition the substrate was kept at room temperature. A custombuilt quartz microbalance was used to measure the deposition rates. Molecular evaporation rates ranging from 0.2 to $0.8 \mathrm{~nm}$ per minute were used. In various exposures, submonolayer coverages down to a few percent of a monolayer have been prepared with high reproducibility. All SubPc coverages refer to the full monolayer of the hexagonal closepacked structure (Sec. III B 2). The error in the thickness of the molecular layers is on the order of 10\%-20\%. By analyzing the molecular layers before and after annealing to 360 $\mathrm{K}$, no characteristic changes could be observed in the STM images as well as in photoelectron spectroscopy experiments.

Figure 1 shows the chemical and geometric structure of the chloro[subphthalocyaninato]boron(III) (SubPc) adsorbate investigated here. According to x-ray diffraction data from the bulk molecular crystal analyzed by Kietaibl, the bond length between the central $\mathrm{B}$ and axial $\mathrm{Cl}$ is $1.8 \AA$ and the distance between the centers of the peripheral benzene rings is $7.6 \AA^{20}$ The geometric and electronic structures of the SubPc molecule have been calculated with the semiemipirical AM1 method as well as with $a b$ initio density functional theory (DFT) ${ }^{22,25}$ We repeated $a b$ initio DFT calculations with the B3LYP exchange-correlation function and the $6-31$ Gd basis set using the GAUSSIAN98 program package. ${ }^{26}$ The effective atomic charges displayed in Fig. 1(c) were determined from the computed electrostatic potential according to the CHELPG option implemented in GAUSSIAN98. These charges are least-squares fitted to reproduce the electrostatic potential at a large number of points within a shell surrounding the molecule. In SubPc an excess of negative charge is found on the electronegative atoms which surround the electron-deficient boron [Fig. 1(c)]. The negative charge is compensated by an electron deficit localized mainly at the six most central carbon atoms. As a consequence the SubPc is a polar molecule with negative charge at the $\mathrm{Cl}$ and posi- tive charge in the conjugated core. This results in a calculated axial permanent dipole moment of $1.0 \mathrm{e} \AA$, which agrees well with the experimental value of $1.1 \mathrm{e} \AA .^{22}$

The molecular arrangements on the substrate were studied by means of a homebuilt STM, operating at room temperature. All STM images were obtained in the constant-current mode by recording the vertical tip movement. Analysis of the electronic structure of the molecular layers were performed by photoelectron spectroscopy in a VG ESCALAB MKII instrument. The XPS experiments were conducted with a nonmonochromatized $\mathrm{Mg} / \mathrm{Al}$ twin anode as an X-ray source. Ultraviolet photoelectron spectroscopy (UPS) was performed using a nonmonochromatized He gas discharge line source. The main line of the lamp is He $I_{\alpha}$ with a photon energy of $21.2 \mathrm{eV}$. The electrons emitted from the sample are detected in a hemispherical $150^{\circ}$ analyzer with three channeltron electron counters.

\section{RESULTS AND DISCUSSION}

Using photoelectron spectroscopy the electronic structure of a sample can be analyzed. With XPS the binding energies of the atomic core levels can be determined. Different chemical environments of an adsorbate, or a species in general, lead to slightly different binding energies of the atomic core levels. This energy difference in the binding energy is called a "chemical shift." 27 UPS is used to obtain information about the density of states (DOS) of the valence band and about the DOS close to the Fermi energy. Consequently, XPS and UPS reveal details of both the adsorbate and substrate electronic structure and therefore of the molecular bonding. These experiments are complemented by series of STM images which provide a real-space map of topographic and electronic features on the atomic scale related to the adsorbate overlayers. Therefore, detailed insight into the geometric and electronic structures of the samples is achieved by the combination of photoelectron spectroscopy and STM, presented below.

\section{A. Adsorption chemistry and electronic structure of SubPc on $\mathrm{Ag}(111)$}

XPS measurements of molecular layers with thickness ranging from submonolayer to multilayer coverage have 


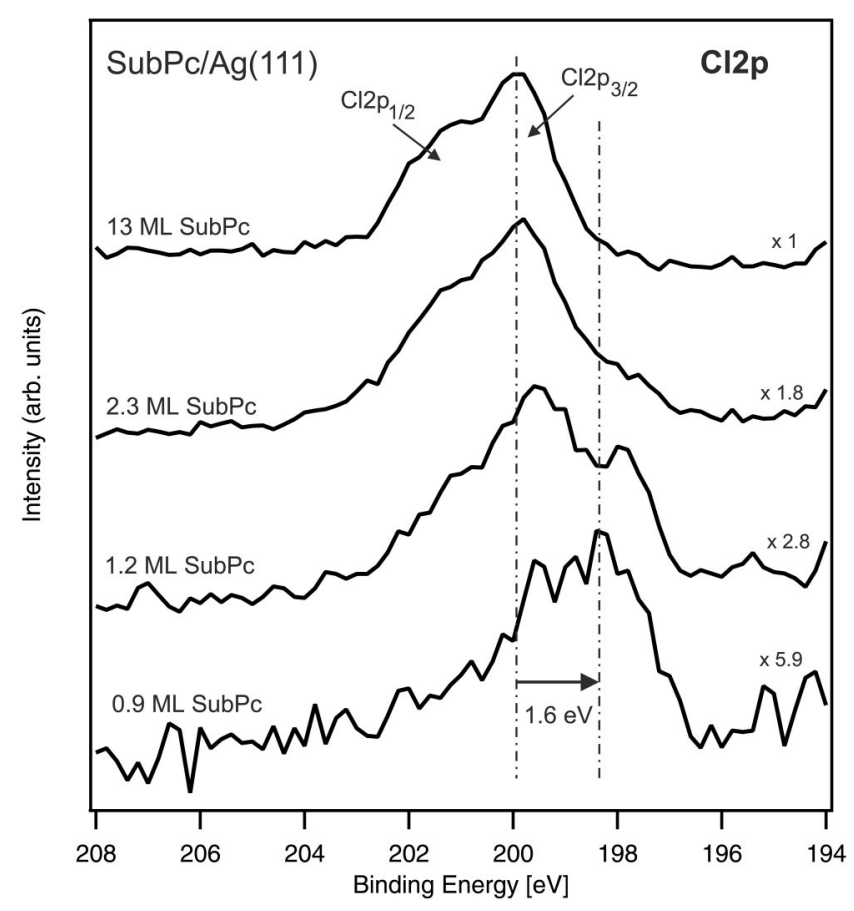

FIG. 2. XPS spectra of the $\mathrm{Cl} 2 p$ peak for increasing SubPc coverage measured with $\mathrm{Mg} K \alpha$ excitation. A chemical shift towards lower binding energy is observed for submonolayer coverage.

been performed. Figure 2 shows the evolution of the $\mathrm{Cl} 2 p$ core level peak for increasing film thickness. A large shift of $1.6 \mathrm{eV}$ towards lower binding energy was observed for the $\mathrm{Cl} 2 p$ peak when changing from a coverage of 13 monolayer (ML) to submonolayer coverage. Due to the surface sensitiv-

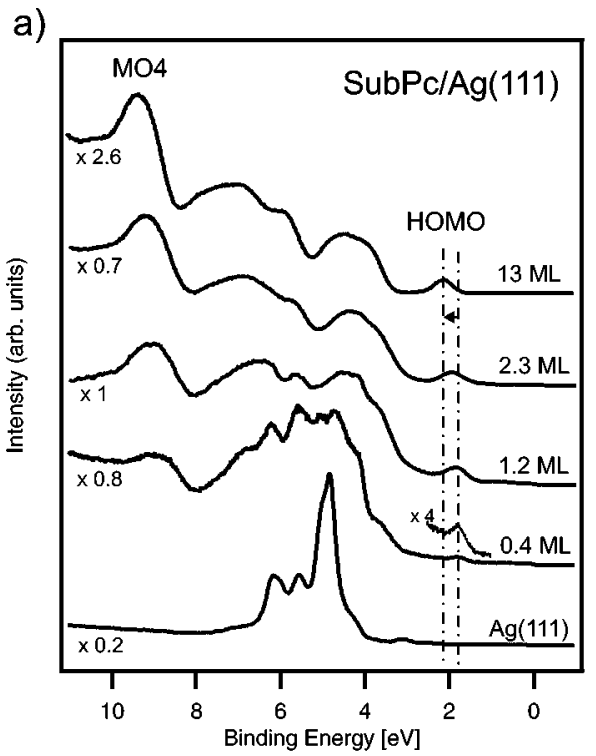

ity of XPS, ${ }^{28}$ the spectrum for $13 \mathrm{ML}$ is dominated by bulklike SubPc features, whereas for submonolayer coverage the XPS measurements are sensitive to the SubPc-Ag interaction. In contrast to the large shift of the $\mathrm{Cl} 2 p$ peak, the $\mathrm{C} 1 s$ and the $\mathrm{N} 1 s$ core level peaks shifted only $0.2-0.3 \mathrm{eV}$ towards lower binding energies for submonolayer coverage. Thus, the observed $\mathrm{Cl} 2 p$ core level shift is interpreted as a "chemical shift" 27 due to the interaction of the $\mathrm{Cl}$ atom of the SubPc with the Ag substrate. The measured binding energy of the $\mathrm{Cl} 2 p$ core level for submonolayer coverage corresponds well to the binding energy obtained for $\mathrm{Cl}$ on $\mathrm{Ag}(110)$ as measured by Briggs and co-workers. ${ }^{29} \mathrm{We}$ therefore conclude that the $\mathrm{Cl}$ in SubPc interacts with the $\mathrm{Ag}$ substrate and the SubPc is adsorbed with the $\mathrm{Cl}$ towards the substrate. Since the peak shifts to lower binding energy, the chemical shift is attributed to a partial electron charge transfer from the $\mathrm{Ag}$ substrate to the $\mathrm{Cl}$ of the SubPc.

Additional information on the SubPc layers was gained by complementary UPS measurements. In Fig. 3 the evolution of the UPS spectra measured with He I excitation is presented for increasing coverage. Besides the highest occupied molecular orbital (HOMO) located at a binding energy of about $E_{B}=1.8 \mathrm{eV}$ (denoted as HOMO in Fig. 3) additional molecular orbitals with higher binding energies are visible. Especially the peak around $E_{B}=9 \mathrm{eV}$ (labeled as MO4 in Fig. 3) is easy to identify even for submonolayer coverage since no features of the $\operatorname{Ag}(111)$ valence band are located in this energy range. Below $1 \mathrm{ML}$ the features of the $\mathrm{Ag}(111)$ substrate contribute significantly to the UPS spectra. Above $1 \mathrm{ML}$ coverage the $\mathrm{Ag}$ features vanish and the peaks associated with SubPc orbitals shift to higher binding energies. However, the shape of the molecular features in these spectra remains almost unchanged. Because of the high surface

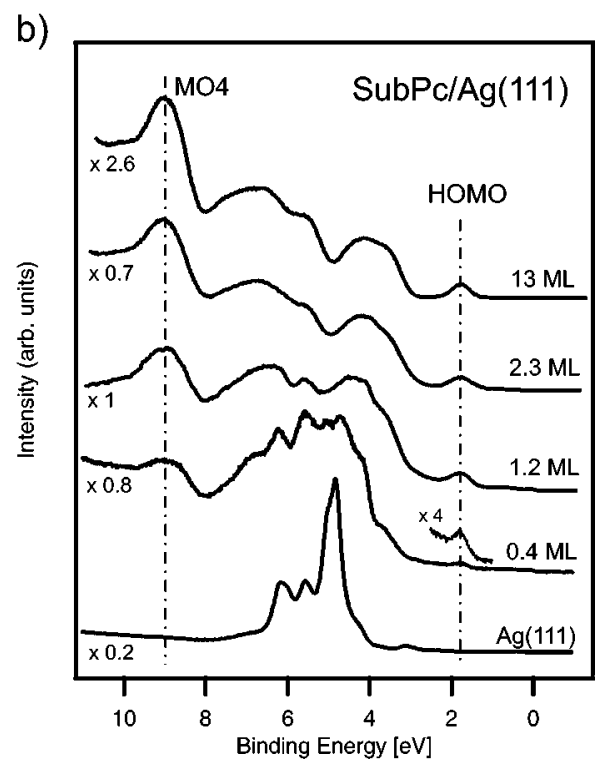

FIG. 3. UPS spectra of the valence band of SubPc on $\mathrm{Ag}(111)$ for increasing SubPc coverage measured with He I excitation. For the clean $\operatorname{Ag}(111)$ substrate, the $\mathrm{Ag} 4 d$ valence states dominate. (a) A shift of the spectra to higher binding energies is observed at higher coverage. (b) The same spectra as in (a) shifted to match the HOMO position for each film thickness. Apart from the disappearance of the Ag features no significant changes are observed with increasing film thickness. In particular, the energy difference between the HOMO and the MO4 is equal for all layers. The binding energies are given with respect to the Fermi energy $E_{F}$ of the substrate. 
TABLE I. Binding energies $\left(E_{B}\right)$ of the HOMO and the MO4 peaks for different SubPc coverage deduced from the UPS spectra and compared to numerical calculations. The experimentally deduced energy difference $\Delta E_{\text {HOMO-MO4 }}$ between the HOMO and the $\mathrm{MO} 4$ is constant, independent of the coverage. For the $[\mathrm{SubPc}]^{+}$a shift of $3.36 \mathrm{eV}$ to higher binding energy and a decrease of $0.36 \mathrm{eV}$ has been calculated for the HOMO and $\triangle E_{\text {НОМо-MO4 }}$, respectively. In the UPS measurements neither such a shift to higher binding energy nor a change in $\Delta E_{\text {HOMO-MO4 }}$ was observed for the layers with submonolayer coverage compared to multilayers. This is a strong evidence against dissociation of SubPc upon adsorption. The binding energies of the UPS measurements are related to the Fermi energy $E_{F}$ of the $\operatorname{Ag}(111)$, whereas the binding energies for the SubPc and $[\mathrm{SubPc}]^{+}$are calculated for the free molecule and therefore related to the vacuum energy $E_{V}$.

\begin{tabular}{lccc}
\hline \hline & $\begin{array}{c}E_{B} \text { HOMO } \\
{[\mathrm{eV}]}\end{array}$ & $\begin{array}{c}E_{B} \mathrm{MO} 4 \\
{[\mathrm{eV}]}\end{array}$ & $\begin{array}{c}\Delta E_{\text {HOMO-MO4 }} \\
{[\mathrm{eV}]}\end{array}$ \\
\hline 0.4 ML SubPc & $1.78 \pm 0.03$ & $8.90 \pm 0.10$ & $7.12 \pm 0.10$ \\
1.2 ML SubPc & $1.83 \pm 0.02$ & $8.98 \pm 0.03$ & $7.14 \pm 0.04$ \\
2.3 ML SubPc & $1.93 \pm 0.02$ & $9.09 \pm 0.03$ & $7.16 \pm 0.04$ \\
13 ML SubPc & $2.16 \pm 0.02$ & $9.27 \pm 0.03$ & $7.11 \pm 0.04$ \\
& & & \\
Calculation SubPc & 5.30 & 12.17 & 6.87 \\
${\text { Calculation [SubPc }{ }^{+}}^{+}$ & 8.66 & 15.17 & 6.51 \\
\hline \hline
\end{tabular}

sensitivity of UPS, ${ }^{28}$ only SubPc molecules with bulk-like interaction are probed in the case of 13 ML. Different occupied molecular orbitals corresponding to bulk SubPc can be distinguished in this spectrum. In Fig. 3(b) the shift of the different spectra is compensated by matching the energy position of the HOMO for all coverages. With increasing film thickness no significant change in the electronic structure of SubPc is observed. In particular, the energy differences $\Delta E_{\text {Hомо-MO4 }}$ between the HOMO and the MO4 are equal within their errors (Table I). This indicates that the frontier orbitals of the $\pi$-electron system appear not to be affected by the charge transfer which is observed in the XPS measurements, as evidenced above. Therefore, the Ag-Cl charge transfer seems to be localized at the $\mathrm{Cl}$, not affecting the extended ring system of the SubPc molecule.

However, there is a shift of the binding energy of the molecular orbitals to higher energies, which needs to be explained. In general, such a shift can occur either due to a charge transfer between the substrate and the adsorbed molecules, due to screening effects of the photoemission hole, ${ }^{30}$ or even due to charging of the molecular layer upon irradiation. In the following we discuss the possible influences of these three mechanisms on our results. Charge transfer: the charge transfer between a substrate and the adsorbate is most pronounced for coverages up to one monolayer. Usually no additional shift is observed for different multilayer coverages due to the localization of the charge transfer to the interface and because of the high surface sensitivity of the UPS measurements. ${ }^{28}$ Therefore, the shift we observed in the UPS spectra by increasing the coverage from 2.3 ML to $13 \mathrm{ML}$ can hardly be explained by a charge transfer between the Ag substrate and the SubPc layers. Screening effects: for a mo- lecular layer in close proximity to a metal, a photoemission hole in the molecular layer is screened by the induced image charge in the metallic substrate. Bulk SubPc is nonconductive, and therefore a photoemission hole created near the surface of a multilayer is less effectively screened than a hole created in a molecular monolayer on a metal substrate. As a consequence, higher binding energies of the molecular orbitals are observed for a thick film compared to a thin film on a metallic substrate. Shifts due to screening of a photoemission hole have, for instance, been described for $\mathrm{C}_{60}$ monolayers on metallic substrates. ${ }^{30,31}$ Irradiation charging: in the case of a nonconductive film, the emitted photoelectrons lead to positive charge at the sample surface. With increasing surface charge densities, the electron binding energies as observed in UPS are shifted by the Coulomb potential. Therefore, the shift we observed in the UPS spectra of increasing multilayer coverage is attributed to a less effective screening of the photoemission hole at higher coverage and to charging of the molecular layer.

There is no evidence for decomposition of the SubPc molecules upon adsorption on the $\operatorname{Ag}(111)$ as only little changes are observed in the UPS spectra for different film thicknesses. Nevertheless, in a "Gedanken experiment" adsorption of SubPc, accompanied by a splitting off of the chlorine, might be a possible process. Indeed, dissociative chemisorption with a sticking coefficient close to unity occurs during the exposure of $\mathrm{Cl}_{2}$ to $\mathrm{Ag}$ substrates at room temperature. ${ }^{29}$ Furthermore, our $a b$ initio DFT calculations revealed that the SubPc molecule without the $\mathrm{Cl}$ is stable as a cation-i.e., as $\mathrm{a}+1 e$ charged ion (denoted in the following as $[\mathrm{SubPc}]^{+}$). The calculations revealed that an energy of $5.6 \mathrm{eV}$ is needed to split the free SubPc molecule in $\mathrm{Cl}^{-}$and $[\mathrm{SubPc}]^{+}$. In a theoretical study of the chlorine adsorption on the $\operatorname{Ag}(111)$ surface the adsorption energy is calculated to be on the order of $3 \mathrm{eV}$ per $\mathrm{Cl}$ atom. ${ }^{32}$ Therefore, the calculations support an intact adsorption of the SubPc molecule on the $\mathrm{Ag}(111)$ surface. According to the calculations of the $[\mathrm{SubPc}]^{+}$, all molecular orbitals shift to higher binding energies compared to SubPc; e.g., the shift of the HOMO is $3.36 \mathrm{eV}$. In the case of the $[\mathrm{SubPc}]^{+}$, we calculated a decrease in the energy difference $\Delta E_{\text {Номо-мO4 }}$ of $360 \mathrm{meV}$. In the UPS spectra neither such a dramatic shift of the spectra to higher binding energy nor a decrease of $\Delta E_{\text {HOMO-MO4 }}$ was observed (Table I). In addition to the theoretical argumentation from above, this is strong experimental evidence against a dissociation of SubPc upon adsorption on $\mathrm{Ag}(111)$.

The work function change of the sample upon SubPc adsorption could also be inferred from UPS measurements. The work function was measured by applying a negative voltage to the sample in order to cover the whole width of the spectrum including the low-energy cutoff of the emitted photoelectrons. The obtained values are listed in Table II showing a decrease of the work function. A linear extrapolation of the values for $0.4 \mathrm{ML}$ and $0.5 \mathrm{ML}$ leads to a decrease in the work function of $\Delta \phi \approx-1 \mathrm{eV}$ for $1 \mathrm{ML}$. Such a reduction of the sample work function is expected for the adsorption of SubPc with the $\mathrm{Cl}$ atom towards the $\mathrm{Ag}$ substrate. In this configuration the permanent dipole moment of the SubPc is perpendicular to the Ag surface, pointing away from it. Therefore, the surface dipole of the metal is reduced which 
TABLE II. Measured work functions of SubPc layers on $\mathrm{Ag}(111)$. A decrease of the work function is observed upon SubPc adsorption.

\begin{tabular}{lcc}
\hline \hline & $\phi[\mathrm{eV}]$ & $\Delta \phi[\mathrm{eV}]$ \\
\hline $\operatorname{Ag}(111)$ & $4.60 \pm 0.02$ & \\
0.4 ML SubPc & $4.22 \pm 0.02$ & $-0.38 \pm 0.03$ \\
0.5 ML SubPc & $4.13 \pm 0.02$ & $-0.47 \pm 0.03$ \\
\hline \hline
\end{tabular}

leads to a reduction of the work function. ${ }^{33}$ According to the simplest model where the dipoles of the adsorbed molecules are treated as a uniform dipole layer, the change of the work function is given by ${ }^{34}$

$$
\Delta \phi=e p N_{A} / \epsilon_{0} .
$$

In this equation $p$ is the molecular dipole moment, $N_{A}$ the number of dipoles per surface area, and $\epsilon_{0}$ the permittivity of free space. Using the SubPc dipole moment of $1.0 \mathrm{e} \AA$, Eq. (1) leads to a decrease of $\Delta \phi=-0.58 \mathrm{eV}$ for $1 \mathrm{ML}$. Screening by surface charges induced in the metallic substrate can be adequately represented by an image dipole, provided that the charge distribution which produces the original dipole little overlaps with the electron density of the substrate. Assuming this to be the case, the image dipole has the same orientation, because the SubPc dipole is perpendicular to the surface. Therefore, the total effective dipole moment per molecule is doubled, ${ }^{33}$ leading to a change in the work function of $\Delta \phi=-2 \times 0.58 \mathrm{eV}=-1.16 \mathrm{eV}$. This value compares with the measured reduction of the work function, $\Delta \phi \approx-1 \mathrm{eV}$. For a more accurate treatment of the calculated work function change, one has to take into account that an opposite electric field is generated at the site of any particular dipole by all the surrounding parallel dipoles. ${ }^{34}$ However, the deviation between the experimental and the calculated effective dipole moment and change in work function could also be affected by the partial charge transfer from the $\mathrm{Ag}$ substrate to the $\mathrm{Cl}$ atom of the SubPc molecule as observed in the XPS measurements. This charge transfer would lead to an induced dipole moment pointing against the surface which would also reduce the effective dipole moment of the adsorbed molecule. In view of the reasonable agreement achieved with the simple equation (1), our work function measurements give additional evidence that the SubPc is adsorbed intact with the $\mathrm{Cl}$ towards the $\mathrm{Ag}(111)$ surface as illustrated in Fig. 4.

\section{B. Two-dimensional superstructures and phase behavior of SubPc on $\operatorname{Ag}(111)$}

While photoelectron spectroscopy experiments provided a detailed picture of the average chemical bonding of the SubPc molecules in a close-to-complete monolayer on $\mathrm{Ag}(111)$, by STM individual molecules in their 2D arrangement can be addressed and analyzed. In time-lapsed imaging sequences slow dynamical processes (msec to $\mathrm{min}$ ) are also accessible. In our STM images of the SubPc layers different overlayer patterns and phases can be distinguished.

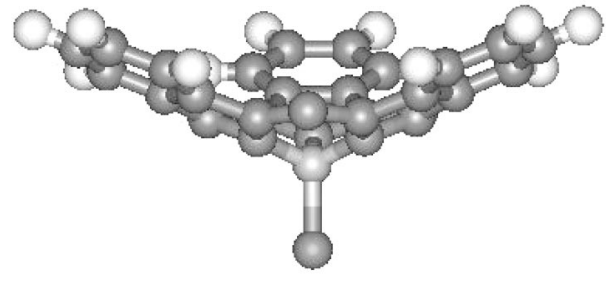

$\operatorname{Ag}(111)$

FIG. 4. Schematic sketch of the inferred adsorption geometry of SubPc on $\mathrm{Ag}(111)$ at monolayer coverage. Each SubPc is adsorbed with the $\mathrm{Cl}$ atom towards the $\mathrm{Ag}$ surface.

\section{Honeycomb pattern: A chiral structure made of achiral molecules}

STM measurements on $\mathrm{Ag}(111)$ with a SubPc coverage of approximately $0.2-0.5$ ML show substrate terraces partially covered by $2 \mathrm{D}$ ordered islands. Within these islands the molecules are assembled in a 2D overlayer with a hexagonal honeycomb pattern (Fig. 5). Clearly, the periodic vacancies which give rise to the low surface packing density of these islands can be observed in these images. The molecules are imaged as protrusions with a height of $\approx 4.5 \AA$ for positive sample bias voltages around $1 \mathrm{~V}$. This apparent height reflects the increased tunneling current due to the presence of molecular levels which are significantly coupled to electronic states of the substrate and the STM tip. In high-resolution STM images individual SubPc molecules are resolved as triangular structures with trefoil shape. In Fig. 5(a) the schematic structures of six SubPc molecules are outlined within the STM image. The intermolecular distance in the honeycomb pattern measured from the STM images is 17.9 $\pm 1.0 \AA$. This leads to a packing density of 0.24 molecules $/ \mathrm{nm}^{2}$ for the $2 \mathrm{D}$ honeycomb pattern.

In the STM experiments domains or islands with two different orientations of the honeycomb pattern with respect to the $\operatorname{Ag}(111)$ substrate have been observed. The angle between these two orientations is $9^{\circ} \pm 1^{\circ}$. This experimental finding - that only two different orientations of the honeycomb pattern exist-implies that besides the intermolecular interaction also molecule-substrate interactions are crucial for establishing the particular registries of the overlayer. In the case of a dominant intermolecular interaction and a negligible molecule-substrate interaction, many more different domain orientations of the overlayer pattern should have been found.

A close inspection of the high-resolution images reveals the orientation of individual SubPc molecules. Each molecule is rotated with respect to the principal axis of the honeycomb pattern. The angle between the phenyl ring and the line joining the center of a molecule with the center of each honeycomb is about $23^{\circ} \pm 5^{\circ}$. In the honeycomb pattern of Fig. 5(a) each SubPc molecule is rotated counterclockwise, while in the pattern of Fig. 5(b) the molecules are rotated clockwise. As a result, the two different orientations of the 

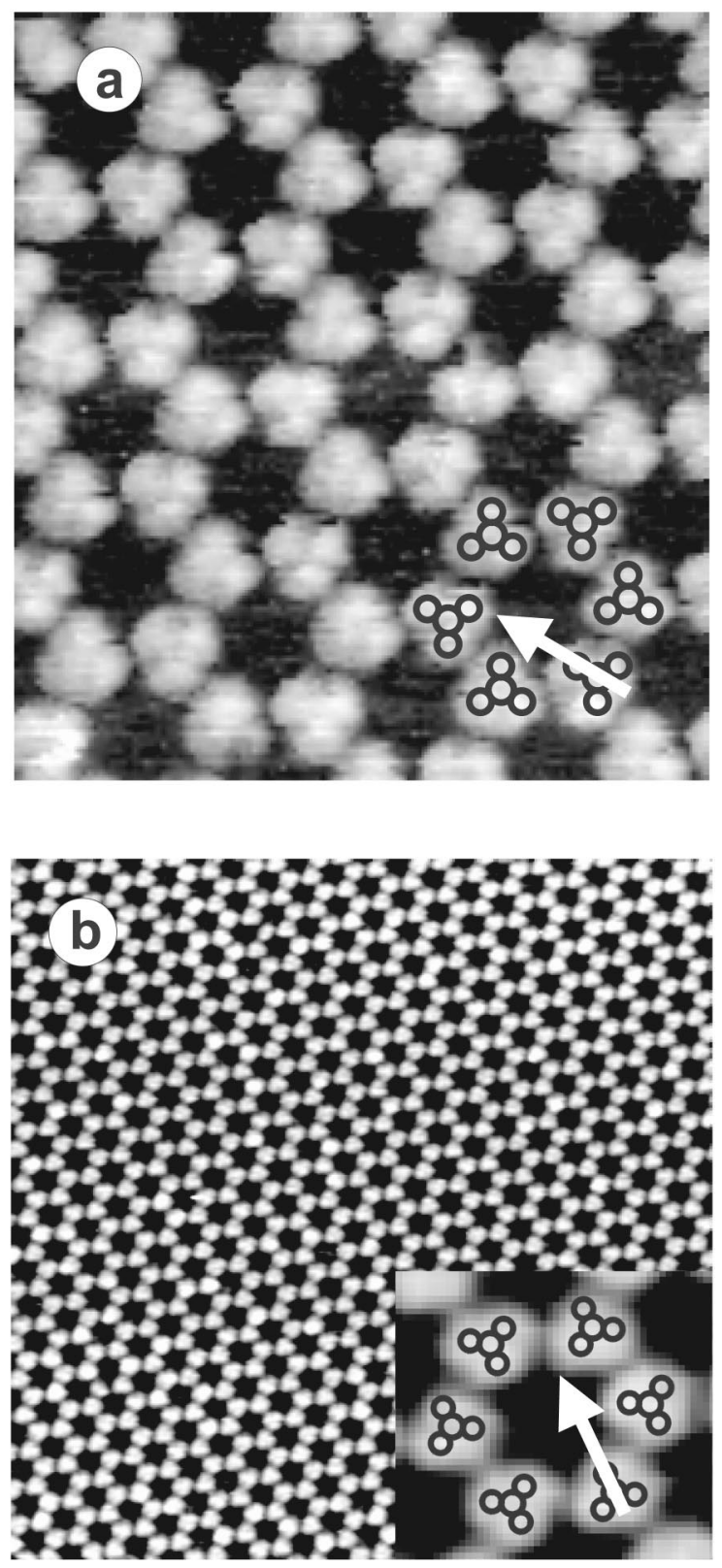

FIG. 5. STM images of the 2D "honeycomb" overlayer of SubPc on $\mathrm{Ag}(111)$. (a) Scan range $14 \times 16 \mathrm{~nm}, I=10 \mathrm{pA}, U$ $=0.7 \mathrm{~V}$. Single molecules are observed with submolecular resolution. The internal structure of the SubPc molecules is outlined at the bottom right (drawn to scale). The dark region in the center of each honeycomb represents the underlying silver substrate. The phenyl rings of the molecules point to the left side of the center of the honeycomb, as indicated by the arrow. (b) Scan range 56 $\times 56 \mathrm{~nm}^{2}, I=10 \mathrm{pA}, U=1.2 \mathrm{~V}$. The observed honeycomb ordering exhibits a very high perfection. The inset shows a magnified image of one "honeycomb." For this orientation of the honeycomb pattern, the phenyl rings of the molecules point to the right side of the center of the honeycomb. Therefore, the two honeycomb structures from (a) and (b) are enantiomorphic.

honeycomb structure have different chirality, although the SubPc molecules and the $\mathrm{Ag}(111)$ surface alone are intrinsically achiral. The 2D self-organized domains or islands have enantiomorphic character.
In order to recognize the interactions responsible for the observed structures, it is instructive to consider possible models of the overlayer structure. However, it was not possible to simultaneously image the atomic structure of the substrate together with the molecular overlayer which would allow us to determine directly the exact adsorption registry. Nevertheless, the experimental data available together with symmetry arguments allow us to propose a feasible model. All molecules in the honeycomb pattern show the same contrast and the same submolecular structure for both orientations. It is therefore reasonable to assume that all molecules are located in equivalent adsorption positions. The superstructure of SubPc on $\operatorname{Ag}(111)$ which best fits the experimental data is depicted in Fig. 6. It has a $\sqrt{111} \times \sqrt{111} R \pm 4.7^{\circ}(2$ $\mathrm{SubPc}$ ) unit cell and the center of each molecule is located above a silver atom (on top site) and the phenyl rings approximately above threefold hollow sites. In this model, each SubPc molecule is rotated by $23.8^{\circ}$ with respect to the lines joining their centers with the center of each honeycomb. The two orientations of the pattern are rotated with respect to the high-symmetry [1 $1 \overline{1} 0]$ direction of the $\mathrm{Ag}(111)$ substrate by $\pm 4.7^{\circ}$. This proposed model agrees very well with the experimental data: The intermolecular distance is $17.7 \AA$ and the angle between the two domains is $9.4^{\circ}$, compared to $17.9 \pm 1.0 \AA$ and $9^{\circ} \pm 1^{\circ}$ in the STM images, respectively. Furthermore, the rotation of each molecule with respect to the honeycomb lattice corresponds to the experimentally observed orientation.

The intermolecular distance of $17.9 \pm 1.0 \AA$ in the case of SubPc on $\mathrm{Ag}(111)$ is larger than the intermolecular distance of $13 \AA$ measured for the square lattice of SubPc on $\mathrm{Cu}(100)$ (Ref. 35) and the distance of $12 \AA$ for SubPc on $\mathrm{Au}(111)$ (Ref. 36). This larger intermolecular distance on $\mathrm{Ag}(111)$ together with geometrical arguments excludes the fact that the rotation of the SubPc molecules with respect to the honeycomb pattern is caused by steric repulsion. On the other hand, through the rotation of the molecules in the SubPc honeycomb pattern the distance between the phenyl rings of two adjacent SubPc molecules is enlarged. This is most likely caused by the repulsive electrostatic interaction between the phenyl rings, since the $\mathrm{H}$ atoms carry positive partial charges, whereas the outer $\mathrm{N}$ atoms of the phthalocyanine macrocycle carry negative partial charges [Fig. 1(c)]. Thus, judging from the charge distribution of the SubPc molecule a rotation of the molecules with respect to the axes of the honeycomb pattern is energetically favored because of the existence of electrostatic intermolecular interactions.

\section{Hexagonal close-packed pattern}

If the coverage is increased to 0.5-0.9 ML, the SubPc molecules self-organize in a 2D hcp pattern (Fig. 7). The intermolecular distance measured from STM images is 18.9 $\pm 1.0 \AA$ and therefore slightly larger than the one of the honeycomb pattern. This leads to a packing density of 0.32 molecules $/ \mathrm{nm}^{2}$ for the hcp pattern which is $35 \%$ higher compared to the honeycomb pattern. Individual molecules are imaged with submolecular resolution as to the honeycomb pattern. Furthermore, for the hcp pattern two orienta- 

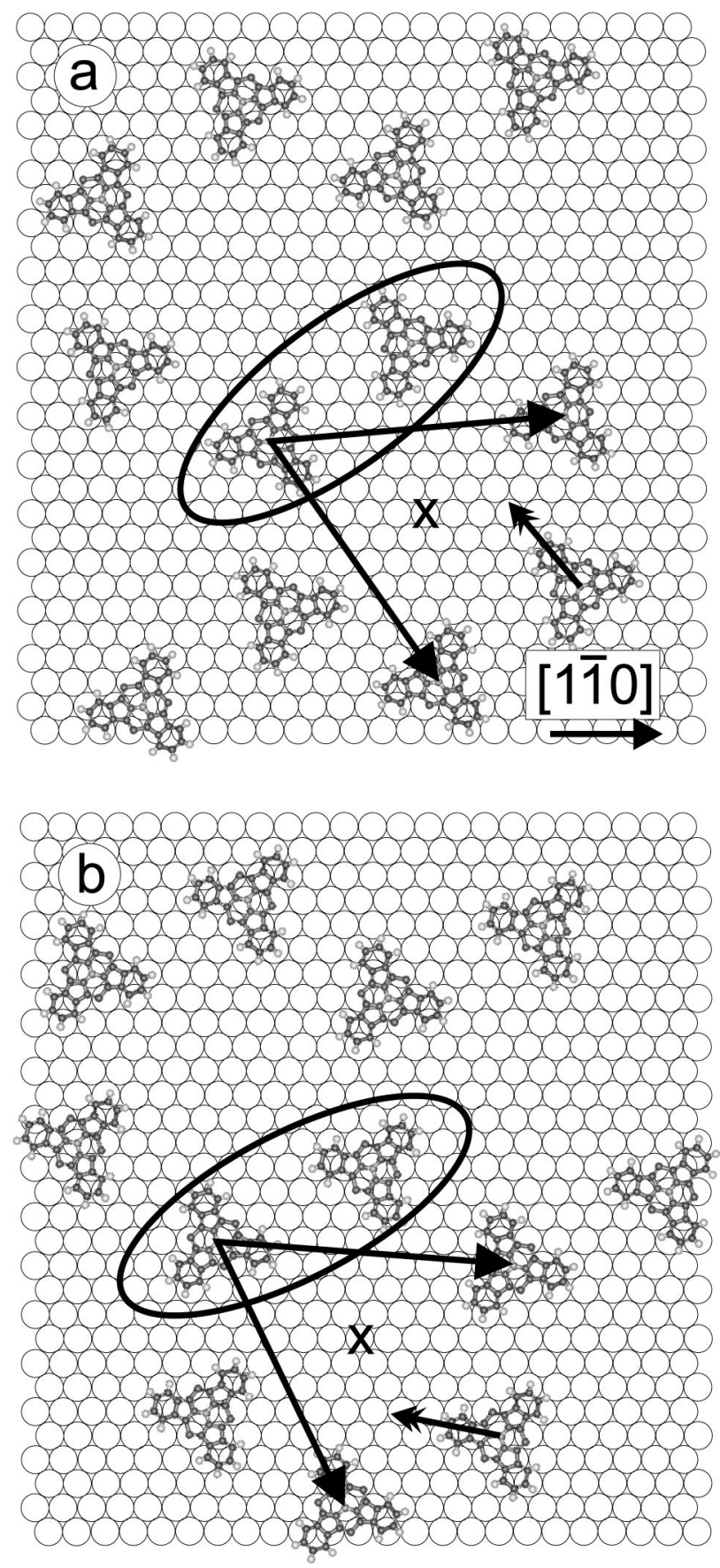

FIG. 6. Proposed model for the honeycomb pattern. The basis consisting of two SubPc and the corresponding Bravais vectors are drawn into the model for both enantiomorphic orientations. The arrow drawn to the molecule at the bottom right indicates the different chirality.

tions with respect to the $\operatorname{Ag}(111)$ substrate were observed as well. The angle between these two orientations is $15^{\circ} \pm 1^{\circ}$.

The difference between the honeycomb and the hcp pattern is more profound than just a missing molecule in each "honeycomb." In contrast to the honeycomb pattern, the SubPc molecules in the hcp pattern are aligned in rows where each molecule is pointing with its phenyl rings to an outer $\mathrm{N}$ atom of the neighboring phthalocyanine. Therefore,
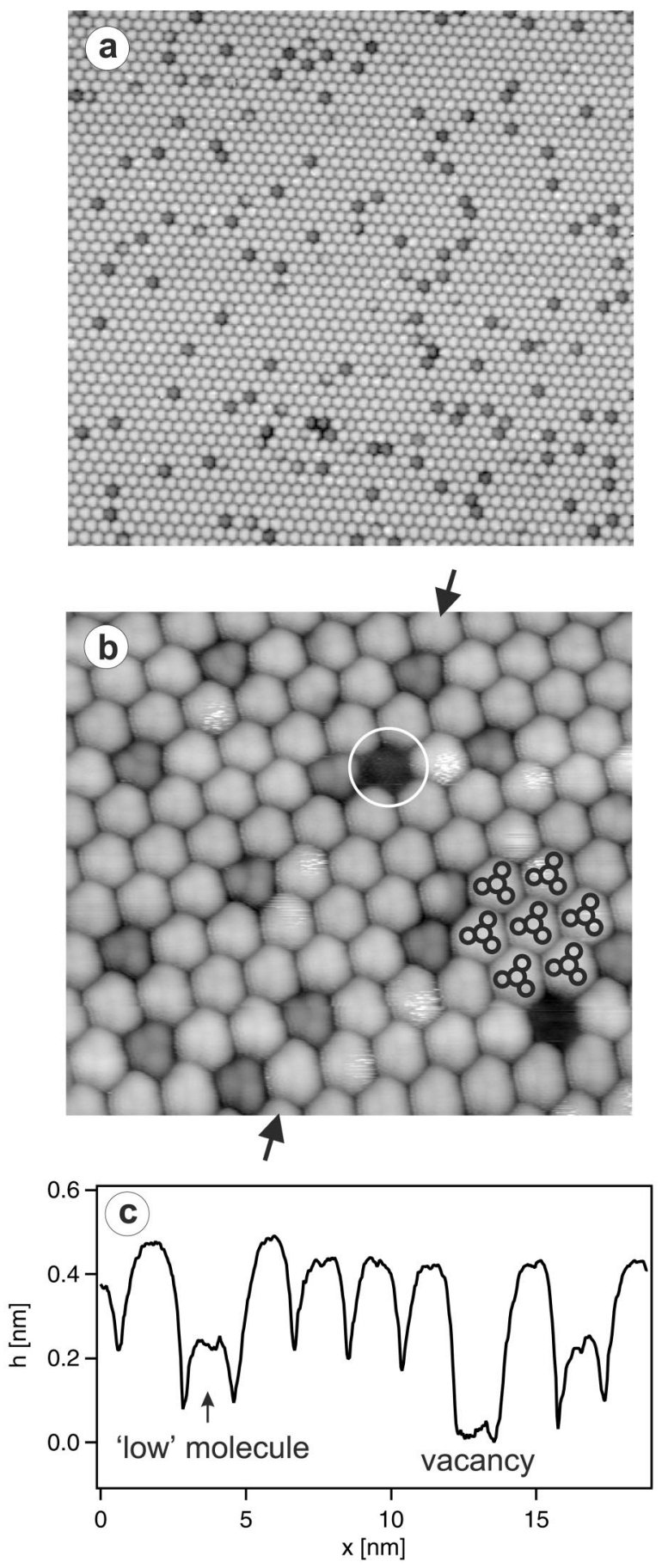

FIG. 7. STM images of the 2D hep overlayer of SubPc on $\mathrm{Ag}(111)$. (a) Scan range $86 \times 82 \mathrm{~nm}^{2}, I=20 \mathrm{pA}, U=1.0 \mathrm{~V}$. A large number of defects is observed in the hcp layer. (b) Zoom image taken with a scan range of $20 \times 18 \mathrm{~nm}^{2}, I=10 \mathrm{pA}, U=1.0 \mathrm{~V}$. Individual SubPc molecules are imaged with submolecular resolution. The internal structure and the orientation of SubPc is outlined on the right-hand side. Two characteristically different defects can be identified. Two vacancy defects are visible; one has been marked by a white circle. The lower apparent height of some molecules is attributed to a local change of their electronic structure, as discussed in the text. (c) Cross section between the two arrows in image (b). The difference in the apparent height for the "low" molecules is clearly visible. 
in the hcp pattern the distance between the phenyl rings of adjacent molecules is increased and the phenyl rings are pointing to the electron-rich outer $\mathrm{N}$ of the phthalocyanine of neighboring molecules. This configuration is expected to be energetically favored due to the charge distribution of the SubPc molecules [Fig. 1(c)] and the resulting electrostatic interaction between adjacent molecules.

Overview images [e.g., Fig. 7(a)] show a surprisingly large number of defects in the hcp layer. However, highresolution STM images [Fig. 7(b)] revealed that these defects are not vacancies, but rather molecules of different apparent height. These molecules have the same shape as the other molecules, but appear in STM at approximately half their height. Therefore, these entities are denoted in the following as "low" molecules. It is expected that these low molecules are different, in the sense that they represent places of lower local density of states (LDOS) within the 2D layer. Consequently, constant-current STM is imaging these molecules at a lower apparent height. ${ }^{37,38}$ The difference in the electronic structure is attributed to a slightly different bonding of the molecule to the Ag substrate. Alternative explanations such as STM image artifacts or chemical modifications in consequence of the sublimation process are considered unlikely for the following reasons. The comparison of the apparent height of the SubPc molecules for the hcp and honeycomb patterns excludes the possibility of a double layer in the images of Fig. 7. The absence of low molecules in the honeycomb pattern gives evidence against a decomposition of the molecules. Furthermore, the amount of low molecules in the hcp pattern is in the order of 5\%-12\%, whereas the purity of the molecules is $>99 \%$. From our experimental data no direct evidence for a reconstruction or local modification of the metal surface was observed upon SubPc adsorption. Since no low molecules are observed in the honeycomb pattern, it is also not likely that the low molecules in the hcp pattern are located on Ag-vacancy sites which would result in topographically lowered molecules. On the other hand, the topographic height difference between low and "normal" SubPc molecules is in the order of a $\operatorname{Ag}(111)$ monoatomic step height. Thus changes in the adsorption mechanism due to the higher molecular coverage cannot be ruled out completely. To analyze the origin of the different electronic structures of the low molecules, it is worth noting that these molecules are slightly rotated with respect to the molecular rows of the hcp pattern. This rotation of the low SubPc molecules along the $\mathrm{B}-\mathrm{Cl}$ axis leads to a slightly different adsorption geometry, which might account for the lower apparent height. However, to gain more insight into this interesting phenomenon more detailed investigations beyond the scope of this paper are needed. In the case of $\mathrm{C}_{60}$ on various metals, different apparent heights for the $\mathrm{C}_{60}$ have been observed. ${ }^{39-42}$ This difference in the apparent height might be due to substrate reconstructions ${ }^{39}$ or due to electronic differences caused by surface interactions, where a mixture of subtle differences in chemical bonding and adsorption geometry takes place. ${ }^{40-43}$

On the basis of the assumption that all molecules within the layer are located at equivalent adsorption sites, a model for the hcp superstructure is proposed. The superstructure of SubPc on $\operatorname{Ag}(111)$ which fits best to the experimental data is
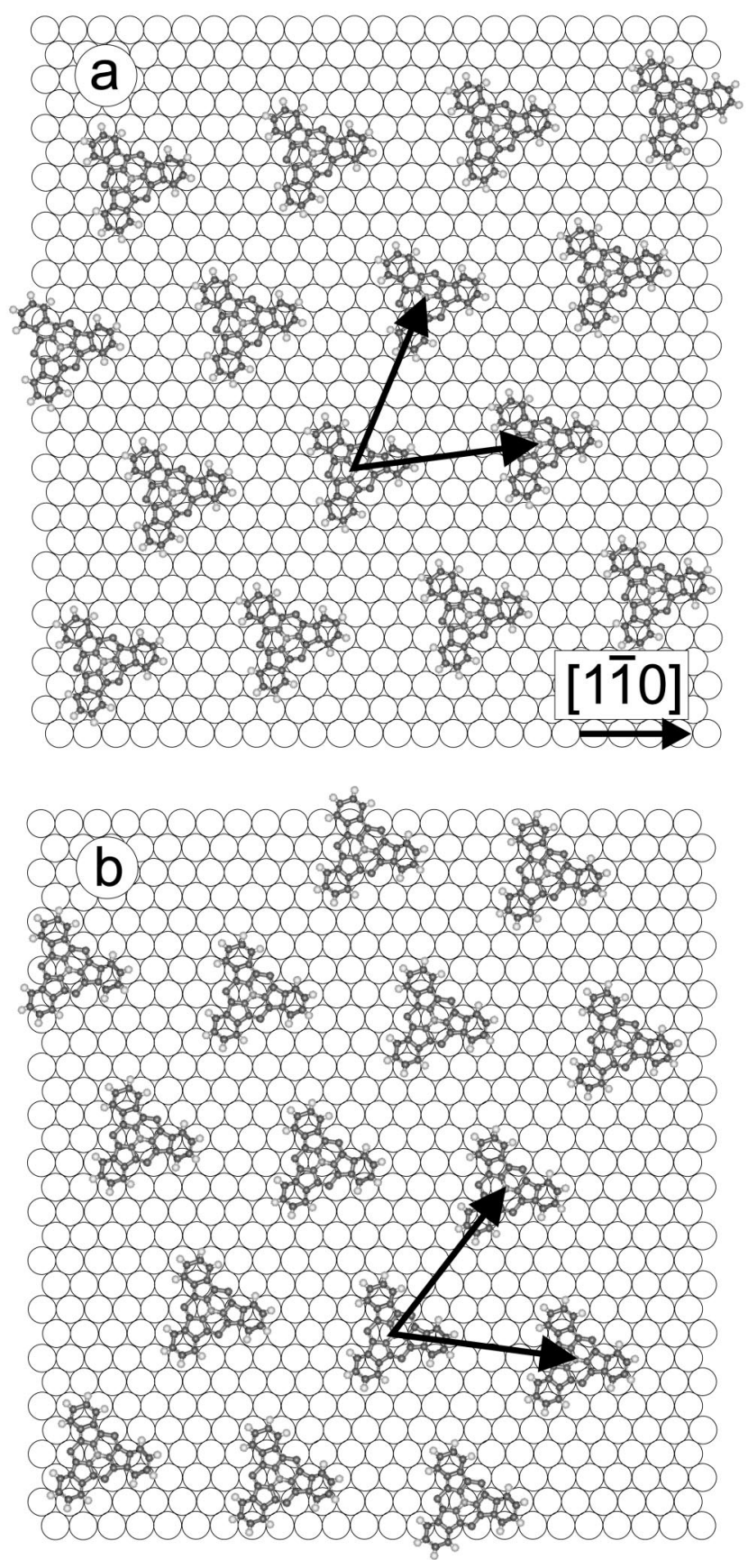

FIG. 8. Proposed model of the hep pattern. The Bravais vectors for the two possible orientations (a) and (b) are indicated.

a $\sqrt{43} \times \sqrt{43} R \pm 7.6^{\circ}$ overlayer as shown in Fig. 8. The two orientations of the pattern are $\pm 7.6^{\circ}$ rotated with respect to the $[1 \overline{1} 0]$ direction of the $\mathrm{Ag}(111)$ substrate. This model is in good agreement with the experimental data: The intermolecular distance is $19.1 \AA$ and the angle between the two domains is $15.2^{\circ}$, while a distance of $18.9 \pm 1.0 \AA$ and an angle of $15^{\circ} \pm 1^{\circ}$ have been measured in the STM images. It is interesting to note that both proposed models for the honeycomb and hcp patterns are consistent because in both models each molecule is located at exactly the same adsorption site: The $\mathrm{Cl}$ on an on-top site and the phenyl rings on hollow sites. 
a
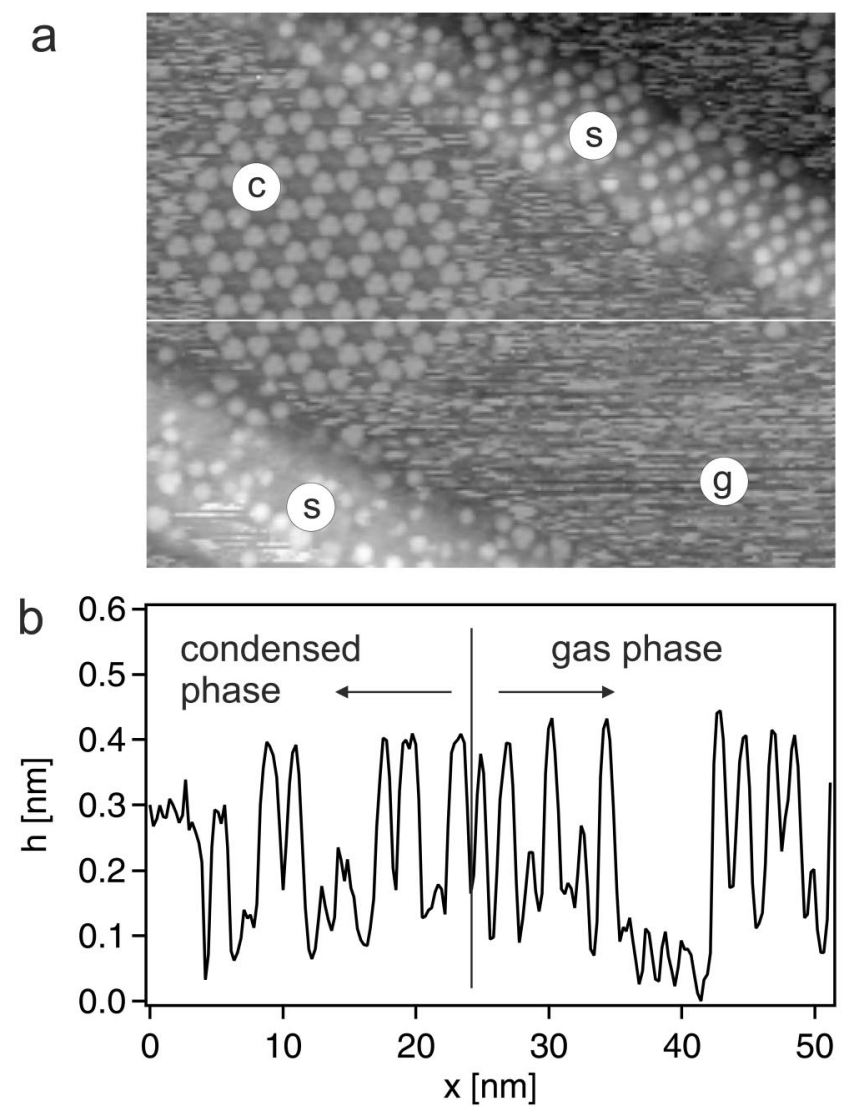

FIG. 9. (a) STM image of SubPc on $\mathrm{Ag}(111)$ with a molecular coverage of $0.3 \mathrm{ML}$ (scan range $51 \times 41 \mathrm{~nm}^{2}, I=12 \mathrm{pA}, U$ $=0.85 \mathrm{~V})$. On the left-hand side of the image a condensed island with a honeycomb pattern (c) is present, whereas on the same terrace next to the condensed island a noisy streak pattern $(\mathrm{g})$ is visible. This noisy pattern is due to mobile molecules which form a $2 \mathrm{D}$ lattice gas. Bunched step edges of the $\mathrm{Ag}(111)$ substrate (s) cross the image at the left bottom corner and in the top right. These steps are decorated by SubPc molecules which form an irregular pattern. The white line represents the location of the scan line shown in (b). (b) Height profile in the fast scanning direction ( $x$ direction). Single molecules are clearly visible and exhibit a characteristic cross section which is similar for molecules in the condensed island and within the regions denoted by the noisy pattern.

A single SubPc molecule has threefold symmetry along the $\mathrm{B}-\mathrm{Cl}$ bond, whereas the $\mathrm{Ag}(111)$ surface has sixfold symmetry. This allows the nucleation of the hcp pattern in four inequivalent domains. For each of the two experimentally observed orientations of the pattern, two orientations differing by a rotation of the molecules of $60^{\circ}$ or equivalently $180^{\circ}$ are possible. These different orientations have been observed experimentally.

\section{Two-dimensional molecular solid-gas equilibrium}

Next to the ordered 2D islands, characteristic "streaky" patterns were observed in our STM data (Fig. 9). From a detailed analysis of individual scan lines, these streaks have been identified as mobile molecules which form a 2D "lattice gas." 44 The molecules in this gas phase are stably adsorbed for a certain time but tend to hop to nearby adsorption sites. The 2D gas phase is observed on all terraces and extends over the whole terrace whether there are nucleated islands or not. Molecular diffusion across step edges is significantly reduced or even completely suppressed as observed in extended time lapse image series. Below a coverage of 0.2 ML the SubPc molecules form a 2D gas phase which coexists with ordered $2 \mathrm{D}$ islands assembling in a honeycomb or a hcp pattern above $0.2 \mathrm{ML}$ or $0.5 \mathrm{ML}$, respectively. The details of this system, which can be described as a 2D thermodynamic equilibrium where molecules are exchanged between the 2D lattice-gas and 2D "solid" phases have been published earlier. $^{44}$

In areas with monoatomic substrate steps bunched together, most of the SubPc molecules are stably adsorbed and remain at their adsorption sites. This leads to the irregularity of the adsorption pattern of the molecules at the step edges (Fig. 9). In addition, these molecules have a slightly different shape in STM images. This may indicate that the chemical or physical bonding of the SubPc at these adsorption sites is different. This is potentially due to the lower coordination and higher chemical reactivity of the step edges, but could also be a consequence of electronic properties of the step edges. ${ }^{45}$

Compared to lower coverage, the hcp pattern is observed in coexistence with mobile molecules forming a $2 \mathrm{D}$ gas phase which appears to have a higher "streak density" than the one coexisting with the honeycomb phase. In general, for increasing molecular coverage, not only the condensed islands increased in size but a higher density of the molecular 2D gas was observed. Therefore, a higher molecular coverage leads to a higher 2D "vapor pressure" of the SubPc molecules and thus to an increase of the condensed area. A very similar behavior was recently observed and analyzed in the case of coadsorption of $\mathrm{N}$ and $\mathrm{O}$ on $\mathrm{Ru}(0001) .{ }^{46}$

\section{Two-dimensional phase behavior}

The different 2D lattice patterns which have been discussed above can be parametrized by the coverage of SubPc molecules: For coverage below $\approx 0.2 \mathrm{ML}$ only mobile molecules forming a $2 \mathrm{D}$ lattice gas are observed. A coexistence of the honeycomb pattern with a 2D lattice gas is observed for coverage on the order of 0.2-0.5 ML, whereas for higher coverage on the order of $0.5-0.9 \mathrm{ML}$ the hep pattern is observed in coexistence with a dense 2D lattice gas. The honeycomb pattern together with the hcp pattern was never observed on the same terrace. For either pattern, honeycomb or hcp, no changes in the pattern were observed upon annealing to $360 \mathrm{~K}$; nor have new patterns appeared. Consequently, we can identify these patterns as two distinctly different 2D solid phases, characterized by their different 2D densities, which are observed in thermodynamic equilibrium with the neighboring lattice gas phase. The molecular density of the 2D gas phase is observed to increase with increasing coverage.

It remains an interesting question to which extent the observed 2D solid and gas phases are thermally excited-i.e., to which extent molecular rotations and vibrations take place. In the case of phosphangulene, a similar cone-shaped molecule like SubPc, some isolated molecules are standing 
upright and are "balancing" on the $\operatorname{Ag}(111)$ surface at a temperature of $6 \mathrm{~K}^{47}$ In the condensed SubPc phases the orientation of individual molecules is visible and molecular rotation is therefore frozen. However, in the SubPc gas phase a rotation of the molecules cannot be ruled out. ${ }^{48}$ In addition, it might be that only the SubPc molecules embedded in a layer are standing upright, whereas isolated molecules on the surface are possibly tilted or wobbling with the $\mathrm{B}-\mathrm{Cl}$ axis to different sides. Internal degrees of freedom of individual SubPc molecules in the gas phase might be hindered with increasing molecular coverage. One could also speculate about correlated motion of nearest-neighbor molecules for a coverage close to one monolayer.

The phase diagrams of 2D lattice gas models have been studied in detail by Monte Carlo simulations for different types of pair and triple interactions. ${ }^{49-52}$ In models with either attractive or repulsive nearest-neighbor interactions, only one ordered phase is observed for either case. ${ }^{49,50}$ Ordered phases with distinctly different symmetries are observed for nearest-neighbor repulsion in conjunction with next-nearest-neighbor attraction and three-body interaction. ${ }^{52}$ In the case of SubPc it is expected that a combination of attractive and repulsive interactions leads to the observed nontrivial phase behavior. In addition to the attractive van der Waals interaction, a repulsive interaction due to the parallel dipole moments of adsorbed molecules is present. Furthermore, there is a delicate interplay of repulsive or attractive interactions depending on the relative angle of two neighboring molecules originating in the inhomogeneous charge distribution. In other words, the interaction between two molecules depends on their relative angle, since the $\mathrm{H}$ atoms of the phenyl rings are charged positive, whereas the outer $\mathrm{N}$ atoms are charged negative [Fig. 1(c)]. Consequently, the phenyl rings repel each other and an electrostatic-favored configuration is obtained if the phenyl rings point to the $\mathrm{N}$ of the neighboring phthalocyanine macrocycle. This tendency is observed for the honeycomb as well as for the hop pattern.

\section{CONCLUSIONS}

SubPc, a large polar molecule with an aromatic $\pi$-electron system, shows a versatile phase behavior with increasing coverage of the first molecular layer grown on $\mathrm{Ag}(111)$. At room temperature, two different rather open self-organized structures, honeycomb and hcp, are observed in coexistence with a 2D lattice gas.

From XPS and UPS measurements in conjunction with calculations of the SubPc molecule it is concluded that the SubPc adsorbs intact with the $\mathrm{Cl}$ atom towards the $\mathrm{Ag}(111)$ substrate. The molecular orbitals of the SubPc molecule are only slightly influenced by adsorption on $\operatorname{Ag}(111)$, allowing the conclusion that the SubPc is essentially physisorbed. The observed electron transfer from the substrate to the $\mathrm{Cl}$ only leads to a small overall shift of the electronic structure of the molecule. This is compatible with the relatively high mobility which enables self-organization and thermodynamic equilibration of different phases at room temperature. In analogy to "self-assembled monolayers" (SAM's), ${ }^{53}$ the molecules spontaneously orient themselves upon adsorption and interaction with the substrate. However, compared to the strong chemisorption of thiol derivatives in SAM, the Ag-Cl bond of SubPc on $\mathrm{Ag}(111)$ is weak. Nevertheless, the $\mathrm{Ag}-\mathrm{Cl}$ bond seems to be crucial for the orientation of the SubPc molecule on the $\operatorname{Ag}(111)$ substrate since it is reported that upon adsorption on $\mathrm{Au}(111)$ the molecules are attached with their isoindolyl groups to the surface. ${ }^{36}$ This characteristic difference most probably originates from the different electronic structures and the resulting different binding forces of the two substrates. Furthermore, the different adsorption geometry could account for the different overlayer structures of SubPc on these substrates.

A very striking feature of the ordered patterns described here is that the molecules are well ordered although the closest atoms of nearest neighbors are separated by about three lattice spacings of the substrate. This excludes any direct chemical interactions, even weak hydrogen bonds like those recently invoked to explain ordered patterns of molecules with a $\pi$-conjugated core adsorbed flat on $\mathrm{Ag}(111)$ (Ref. 54) or $\mathrm{Au}(111)$ (Refs. 9, 54 and 55). Nevertheless, the arrangement of the molecules in the honeycomb and the hcp pattern can be related to the charge distribution of the individual SubPc molecule and the resulting electrostatic interaction. The balance between intermolecular interactions, moleculesubstrate interactions, and thermal energy governs the diffusion of single molecules, island diffusion, nucleation, and self-organization in general. All these effects have been observed by analyzing the behavior of SubPc on $\mathrm{Ag}(111)$. This is a unique possibility to study the delicate interplay of intermolecular and molecule-substrate interactions at work.

\section{ACKNOWLEDGMENTS}

We thank H. Kliesch and D. Wöhrle (University of Bremen, Germany) for synthesizing and providing the SubPc molecules. Helpful discussions with A. Alkauskas, S. Schintke, M. von Arx (University of Basel, Switzerland), and H. Yanagi (Kobe University, Japan) are gratefully acknowledged. Financial support from the Swiss National Science Foundation, the Swiss Top Nano 21 program, the NCCR on Nanoscale Science, and the University of Basel is gratefully acknowledged.

\footnotetext{
*Electronic address: simon.berner@unibas.ch

${ }^{1}$ C. Joachim, J. Gimzewski, and A. Aviram, Nature (London) 408, 541 (2000).

${ }^{2}$ R. Carroll and C. Gorman, Angew. Chem., Int. Ed. Engl. 41, 4378 (2002).

${ }^{3}$ J. Burroughes, D. Bradley, A. Brown, R. Marks, M. Mackay, R.
}

Friend, P. Burns, and A. Holmes, Nature (London) 347, 539 (1990).

${ }^{4}$ H. Katz, J. Mater. Chem. 7, 369 (1997).

${ }^{5}$ R. Gomer, Rep. Prog. Phys. 53, 917 (1990).

${ }^{6}$ J. Venables, Introduction to Surface and Thin Film Processes (Cambridge University Press, Cambridge, England, 2000). 
${ }^{7}$ H. Brune, Surf. Sci. Rep. 31, 121 (1998).

${ }^{8}$ J.V. Barth, Surf. Sci. Rep. 40, 75 (2000).

${ }^{9}$ T. Yokoyama, S. Yokoyama, T. Kamikado, Y. Okuno, and S. Mashiko, Nature (London) 413, 619 (2001).

${ }^{10}$ J.V. Barth, J. Weckesser, G. Trimarchi, M. Vladimirova, A. de Vita, C. Cai, H. Brune, P. Günter, and K. Kern, J. Am. Chem. Soc. 124, 7991 (2002).

${ }^{11}$ D. Walba, F. Stevens, N.A. Clark, and D.C. Parks, Acc. Chem. Res. 29, 591 (1996).

${ }^{12}$ R. Viswanathan, J.A. Zasadzinski, and D.K. Schwartz, Nature (London) 368, 440 (1994).

${ }^{13}$ F. Charra and J. Cousty, Phys. Rev. Lett. 80, 1682 (1998).

${ }^{14}$ Phthalocyanines: Properties and Applications, edited by C. C. Leznoff and A. B. P. Lever (VCH, New York, 1989), Vol. 1-4.

${ }^{15}$ J.K. Gimzewski, E. Stoll, and R.R. Schlittler, Surf. Sci. 181, 267 (1987)

${ }^{16}$ P.H. Lippel, R.J. Wilson, M.D. Miller, C. Wöll, and S. Chiang, Phys. Rev. Lett. 62, 171 (1989).

${ }^{17}$ T. Shimada, K. Hamaguchi, A. Koma, and F. Ohuchi, Appl. Phys. Lett. 72, 1869 (1998).

${ }^{18}$ L. Lozzi, L. Ottaviano, and S. Santucci, Surf. Sci. 470, 265 (2001).

${ }^{19}$ A. Meller and A. Ossko, Monatsch. Chem. 103, 150 (1972).

${ }^{20}$ H. Kietaibl, Monatsch. Chem. 105, 405 (1974).

${ }^{21}$ M.K. Engel, J. Yao, H. Maki, H. Takeuchi, H. Yonehara, and C. Pac, Rep. Kawamura Inst. Chem. Res. 9, 53 (1998).

${ }^{22}$ V.R. Ferro, L.A. Poveda, R.H. González-Jonte, J.M. Garcia de la Vega, T. Torres, and B. Del Rey, J. Porhpyrins Phthalocyanines 4, 611 (2000).

${ }^{23}$ C.G. Claessens, D. González-Rodríguez, and T. Torres, Chem. Rev. (Washington, D.C.) 102, 835 (2002).

${ }^{24}$ A.A. Baski and H. Fuchs, Surf. Sci. 313, 275 (1994).

${ }^{25}$ H. Yanagi, D. Schlettwein, H. Nakayama, and T. Nishino, Phys. Rev. B 61, 1959 (2000).

${ }^{26}$ M. J. Frisch et al., Computer code GAUSSIAN 98, revision A.7, (Gaussian Inc., Pittsburgh, 1998).

${ }^{27}$ S. Hüfner, Photoelectron Spectroscopy, 2nd ed. (Springer-Verlag, Berlin, 1996).

${ }^{28}$ M.P. Seah and W.A. Dench, Surf. Interface Anal. 1, 2 (1979).

${ }^{29}$ D. Briggs, R.A. Marbow, and R.M. Lambert, Chem. Phys. Lett. 53, 462 (1978).
${ }^{30}$ R. Hesper, L.H. Tjeng, and G.A. Sawatzky, Europhys. Lett. 40, 177 (1997).

${ }^{31}$ M.R.C. Hunt, P. Rudolf, and S. Modesti, Phys. Rev. B 55, 7882 (1997).

${ }^{32}$ K. Doll and N.M. Harrison, Phys. Rev. B 63, 165410 (2001).

${ }^{33}$ A. Zangwill, Physics at Surfaces (Cambridge University Press, Cambridge, England, 1988).

${ }^{34}$ H. Lüth, Surfaces and Interfaces of Solid Materials, 3rd ed. (Springer, Berlin, 1995).

${ }^{35}$ H. Yanagi, K. Ikuta, H. Mukai, and T. Shibutani, Nano Lett. 2, 951 (2002).

${ }^{36}$ S. Mannsfeld, H. Reichhard, and T. Fritz, Surf. Sci. 525, 215 (2003).

${ }^{37}$ J. Tersoff and D.R. Hamann, Phys. Rev. Lett. 50, 1998 (1983).

${ }^{38}$ J. Tersoff and D.R. Hamann, Phys. Rev. B 31, 805 (1985).

${ }^{39}$ M. Grobis, X. Lu, and M.F. Crommie, Phys. Rev. B 66, 161408(R) (2002).

${ }^{40}$ E.I. Altman and R.J. Colton, Surf. Sci. 279, 49 (1992).

${ }^{41}$ E.I. Altman and R.J. Colton, Surf. Sci. 295, 13 (1993).

${ }^{42}$ E. Giudice, E. Magnano, S. Rusponi, C. Boragno, and U. Valbusa, Surf. Sci. 405, L561 (1998).

${ }^{43}$ R. Fasel, P. Aebi, R.G. Agostino, D. Naumović, J. Osterwalder, A. Santaniello, and L. Schlapbach, Phys. Rev. Lett. 76, 4733 (1996).

${ }^{44}$ S. Berner, M. Brunner, L. Ramoino, H. Suzuki, H.-J. Güntherodt, and T.A. Jung, Chem. Phys. Lett. 348, 175 (2001).

${ }^{45}$ K. Wandelt, Surf. Sci. 251/252, 387 (1991).

${ }^{46}$ C. Nagl, R. Schuster, S. Renisch, and G. Ertl, Phys. Rev. Lett. 81, 3483 (1998).

${ }^{47}$ R. Lin, K.-F. Braun, H. Tang, U.J. Quaade, F.C. Krebs, G. Meyer, C. Joachim, K.-H. Rieder, and K. Stokbro, Surf. Sci. 477, 198 (2001).

${ }^{48}$ J.K. Gimzewski, C. Joachim, R.R. Schlittler, V. Langlais, H. Tang, and I. Johannsen, Science 281, 531 (1998).

${ }^{49}$ C. Uebing and R. Gomer, J. Chem. Phys. 95, 7626 (1991).

${ }^{50}$ C. Uebing and R. Gomer, J. Chem. Phys. 95, 7636 (1991).

${ }^{51}$ C. Uebing and R. Gomer, J. Chem. Phys. 95, 7641 (1991).

${ }^{52}$ K. Binder and D.P. Landau, Surf. Sci. 108, 503 (1981)

${ }^{53}$ A. Ulman, Chem. Rev. 96, 1533 (1996).

${ }^{54}$ J. Weckesser, A.D. Vita, J. Barth, C. Cai, and K. Kern, Phys. Rev. Lett. 87, 096101 (2001).

${ }^{55}$ M. Böhringer, K. Morgenstern, W.-D. Schneider, R. Berndt, F. Mauri, A.D. Vita, and R. Car, Phys. Rev. Lett. 83, 324 (1999). 